\title{
Efecto del foco atencional sobre el aprendizaje de las habilidades deportivas individuales Effect of attentional focus on the learning of individual sports skills

\author{
Pablo Camacho Lazarraga
} \\ Centro Universitario San Isidoro, Sevilla (España)
}

Resumen. La focalización de la atención de un sujeto puede tener una importante influencia en el aprendizaje y el rendimiento motor de una habilidad deportiva, viéndose ésta condicionada en gran medida por el tipo de retroalimentación suministrada. Numerosos estudios han demostrado que centrar la atención en los efectos del movimiento favorece un desempeño inmediato eficaz. Pero también se han realizado intervenciones con diferentes resultados. El objetivo del presente estudio es revisar y evaluar sistemáticamente la literatura relacionada con la estrategia de focalización externa e interna para el aprendizaje de una habilidad deportiva individual. Se consultaron las bases de datos Web of Science, Scopus, SportDiscus With Full Text y PsycInfo, además de otras fuentes de investigación. Se revisaron los títulos, resúmenes y textos completos. Se seleccionaron 37 estudios que cumplían con los criterios de inclusión y exclusión para su elegibilidad. Los resultados indican que, en líneas generales, reducir la cantidad de información que se le suministra a los sujetos beneficia el aprendizaje y la retención de una habilidad deportiva, ya que la carga atencional disminuye considerablemente, permitiendo con ello disponer de mayores recursos durante su ejecución. Las principales variables moderadoras de los resultados son el tipo de habilidad, el grado de dificultad de la tarea, la edad y el nivel de pericia de los participantes. Estas conclusiones deberían analizarse con cautela, debido al escaso número de artículos localizados y que no podemos asegurar que la estrategia de focalización seguida por los sujetos haya sido únicamente la inicialmente establecida.

Palabras clave. Retroalimentación, instrucciones, focalización externa, focalización interna, aprendizaje motor.

Abstract. An individual's attention focus can have can have an important influence on the learning and performance of a sports skills seeing it conditioned to a large extent by the type of feedback provided. Numerous studies have shown that focusing attention on the effects of movement favors effective immediate performance. But there have also been interventions with different results. The objective of the present study is to review and systematically evaluate the literature related to both internal and external strategies for targeting one's learning of an individual sport skill. The Web of Science, Scopus, SportDiscus With Full Text and PsycInfo databases were consulted, as well as other research sources. The titles, abstracts and complete texts were reviewed. We selected 37 studies that met the inclusion and exclusion criteria for eligibility. The results indicate that, in general terms, reducing the amount of information provided to the subjects benefits learning and the retention of a sports skill, since the attentional load decreases considerably, thereby allowing subjects to have greater resources during its execution. The main moderating variables of the results are the type of skill, the degree of difficulty of the task, the age and the level of expertise of the participants. These conclusions should be analyzed with caution, due to the limited number of localized articles and to the fact that we cannot assure that the targeting strategy followed by the subjects was the one initially established.

Keywords. Feedback, instructions, external focus, internal focus, motor learning.

\section{Introducción}

En los últimos años ha habido un número considerable de estudios en los que se ha demostrado que la focalización de la atención de un sujeto puede tener una importante influencia en el aprendizaje y el rendimiento motor (Golden, 2018; 2001; Land, Frank \& Schack, 2014 Schoenfeld, Vigotsky, Contreras \&; Wulf, 2013; Wulf, Shea \& Park), viéndose éstos en ocasiones deteriorados en condiciones de estrés o límite de tiempo (González, Valdivia-Moral, Cachón, Zurita \& Romero-Ramos, 2017; Jackson, Ashford \& Norsworthy, 2006; Kais \& Raudsepp, 2005; Maynard \& Howe, 1987; Núñez \& García, 2017). Es por ello por lo que, con objeto de optimizar las situaciones de aprendizaje, se han diseñado intervenciones en las que se manipulaba la focalización de la atención de los sujetos a través del tipo de información que se les suministraba (Lohse, Jones, Healy \& Sherwood, 2014; Tse \& Ginneken, 2017), utilizada para dirigir su atención hacia las fuentes de información más relevantes durante el proceso de búsqueda y selección (Sánchez \& Sebastián, 2015).

Autores como Wulf, Chiviacowsky, Schiller \& Gentilini (2010) y Zachry, Wulf, Mercer \& Bezodis (2005) afirman que la eficacia del aprendizaje de las habilidades motrices se ve afectada en gran medida por el tipo de retroalimentación, siendo éste considerado en ocasiones limitador durante el proceso de búsqueda de los estímulos que subyacen del entorno (Newell, 1986).

El término asfixia bajo presión se ha utilizado para definir un rendimiento inferior a pesar del esfuerzo realizado, o los refuerzos ofrecidos para obtener un rendimiento superior (Baumeister \& Showers, 1986). Son muchos los estudios que han tratado de explicar las causas que provocan el deterioro del rendimiento bajo presión en el contexto

Fecha recepción: 21-04-18. Fecha de aceptación: 07-11-18 Pablo Camacho Lazarraga

pcamacholazarraga@gmail.com deportivo (Baumeister, 1984; Beilock \& Carr, 2001; Masters, Polman \& Hammond, 1993; Reeves, Tenenbaum \& Lidor, 2007), siendo dos los principales modelos atencionales propuestos: (1) la teoría de la distracción, que afirma que la presión provoca un cambio de atención hacia las señales irrelevantes, creando esto un entorno de doble tarea que consume y limita la capacidad de la memoria de trabajo tan importante para el desempeño eficaz (Beilock \& Carr, 2001), y (2) la teoría del control explícito o autoenfoque, que afirma que la presión provoca que el sujeto aumente su autoconciencia acerca de cómo realizar correctamente la secuencia del movimiento, dirija la atención hacia el control, altere su carácter automático y como consecuencia interrumpa su desempeño (Baumeister, 1984). Debido a que la conciencia no contiene todo el conocimiento de dichas habilidades, irónicamente se reduce su fiabilidad y éxito durante la actuación. Esta teoría es consistente con la hipótesis de progresión-regresión en la adquisición de habilidades perceptivo-motrices de Fuchs (1962), que postula que actuar en condiciones de estrés puede favorecer una regresión a las primeras etapas de la adquisición de la habilidad, caracterizada por la dependencia a las señales verbales y el conocimiento explícito.

Con el objetivo de evitar la degradación del rendimiento de las habilidades bajo presión, Wulf, Höß \& Prinz (1998) proponen la hipótesis de la acción restringida, que defiende que una estrategia de enfoque externo (EFE), centrarse en los efectos del movimiento, favorece un control automático de la habilidad, y por tanto un desempeño inmediato más eficaz, mientras que una estrategia de enfoque interno (EFI), centrarse en los propios movimientos, interrumpe los procesos de control automático que controlan las acciones de un individuo con eficacia y eficiencia, permitiendo que los procesos inconscientes y rápidos controlen los movimientos.

Una estrategia de orientación cuyo efecto origine una focalización de la atención del sujeto hacia los efectos del movimiento (EFE) se ha demostrado beneficioso para el aprendizaje y el rendimiento en numerosos estudios, como los realizados en golf por Brocken, Kal \& Van der 
Kamp (2016), gimnasia por Abdollahipour, Wulf, Psotta \& Palomo (2015) y lanzamiento de dardos por Lohse, Sherwood \& Healy (2014), debido presumiblemente a que este tipo de enfoque promueve la conectividad funcional, contribuye al acoplamiento meta-acción (Wulf, 2015) y reduce el conocimiento explícito acerca de cómo ejecutar la habilidad, permitiendo la activación del proceso de auto-organización implícito durante la ejecución de las habilidades (Al-Abood, Bennett, Hernandez, Ashford \& Davids, 2002), no generando con ello una gran carga mental, debido a que el procesamiento inconsciente no depende de la memoria de trabajo, y por tanto las demandas que resulten de los requisitos de las tareas múltiples probablemente no sobrecargue y perturbe el rendimiento (Masters, Poolton, Maxwell \& Raab, 2008). Este tipo de estrategia tiene una gran similitud con la enseñanza por analogía, en la que se involucran mecanismos de comportamiento motor similares a los activados durante el procesamiento incidental (Liao \& Masters, 2001). Sin embargo, también ha habido estudios con resultados diferentes, como el de Tse \& Ginneken (2017), en el que se desarrolló una tarea de lanzamiento de dardos, obteniendo resultados favorables al grupo de focalización hacia los propios movimientos (EFI).

Por tanto, y teniendo en cuenta que uno de los factores moderadores de los resultados de los estudios realizados es el tipo de habilidad desarrollada (Raab, 2003), y con la intención de acotar el tema-objeto de este estudio, el objetivo que se plantea es el de revisar y evaluar sistemáticamente la literatura relacionada con la EFE y EFI durante la ejecución de habilidades deportivas individuales, analizando si benefician o perjudican el aprendizaje o el rendimiento.

\section{Método}

\section{Estrategia de búsqueda}

Debido a la heterogeneidad en las medidas de los resultados utilizadas en los estudios que finalmente cumplían con los criterios de inclusión-exclusión, no se realizó ningún metaanálisis (Ioannidis, Patsopoulos \& Rothstein, 2008), como en el estudio realizado por Holfelder \& Schott (2014). En su lugar, se realizó una revisión sistemática de la literatura científica.

Con objeto de identificar todos los estudios relacionados con la enseñanza de una habilidad deportiva a través de la utilización de una EFE y EFI, se realizó una revisión exhaustiva empleando varios procesos de búsqueda de fuentes de investigación: (a) Consulta en cuatro bases de datos electrónicas: Web of Science, Scopus, SportDiscus With Full Text y PsycInfo (hasta el 04/03/2018). Para la selección de los descriptores de búsqueda («feedback», «external focus», «internal focus», «motor learning») se consultaron tanto los Thesaurus de dichas bases de datos como a expertos en la materia. Se realizó una combinación de dichos descriptores para la configuración de la frase de búsqueda. (b) Consulta directa en revistas especializadas sobre revisiones sistemáticas (Annual Review of Psychology, Exercise and Sport Science Reviews, International Review of Sport \& Exercise Psychology, y Psychological Review), (c) consulta de Tesis Doctorales (Proquest Dissertation \& Theses Full Text y Teseo), (d) revisión ascendente de la literatura recuperada, con el fin de localizar investigaciones no identificadas durante el proceso de búsqueda, y (e) literatura gris, referida a documentos no publicados o publicados a través de canales poco convencionales (bases de datos PsycExtra y SIGLE).

\section{Selección de los estudios}

Se seleccionaron de las referencias localizadas aquellos estudios pertenecientes a las áreas temáticas más estudiadas en este campo, aplicándoseles los siguientes criterios de inclusión y exclusión con objeto de asegurar que los límites de la revisión se definieran claramente, y que la estrategia de búsqueda identificara toda la literatura relevante para los objetivos de la revisión (Smith, 2010): (a) artículo original publicado en su totalidad, (b) revisado por pares, (c) relacionado con la temáticaobjeto de estudio y (d) estudio experimental. Se revisaron los títulos y los resúmenes de los estudios localizados, y en los casos necesarios, se realizó un análisis completo de los textos de los estudios resultantes.
Para la aplicación de estos criterios, tres miembros del equipo de investigación revisaron los textos de forma independiente. Finalmente, fueron consensuadas las decisiones derivadas de ello.

\section{Resultados}

\section{Búsqueda y selección de la literatura}

Se localizaron 654 documentos potencialmente relevantes (figura 1). Se eliminaron los duplicados ( $\mathrm{n}=262$ ), reduciéndose a un total de 392 documentos. Se excluyeron aquellos que no cumplían con los criterios de selección establecidos inicialmente tras la revisión de los títulos y resúmenes ( $\mathrm{n}=298$ ) y de los textos completos ( $\mathrm{n}=57)$, siendo finalmente 37 los estudios seleccionados realizados entre los años 1998 y 2017 en los que se desarrolló la EFE y EFI, con una población total de 1193 sujetos a analizar, una media de edad de 21,19 años y diferentes niveles depericia.

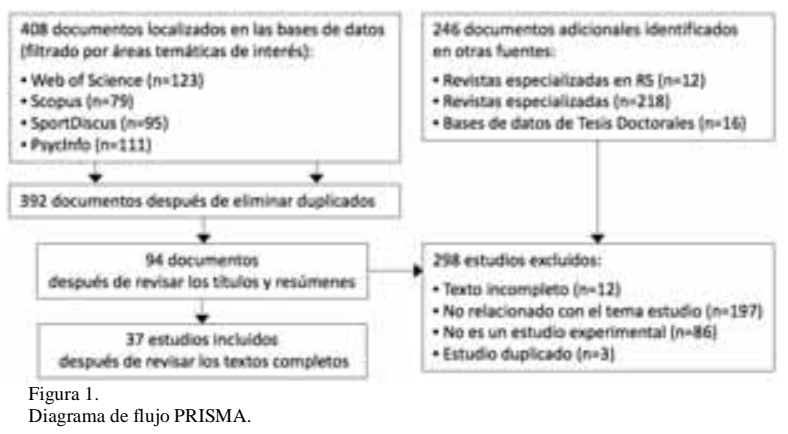

\section{Análisis de los efectos de las variables moderadoras}

En la tabla 1 se muestra una síntesis de los principales resultados de estos estudios.

Se evaluó el efecto de la influencia de diferentes feedback sobre la adquisición, transferencia y retención de determinadas habilidades motrices específicas. Las variables dependientes analizadas fueron la precisión, el tiempo y la cinemática de los movimientos. La duración de las intervenciones y el número de ensayos varió desde una sesión y sólo 16 ensayos (Maxwell \& Masters, 2002) hasta tres sesiones y 540 ensayos (Land et al., 2014). Analizamos los resultados teniendo en cuenta el tipo de habilidad, el grado de dificultad, la edad y el nivel de experiencia de los participantes.

En todas las tareas en las que se analizaba la precisión en los lanzamientos de dardos en sujetos con nivel de pericia bajo, el grupo EFE obtenía los mejores resultados, excepto en el estudio realizado por Emmanuel, Jarus \& Bart (2008), en el que los grupos EFE y EFI mostraban igual desempeño, y en el estudio realizado por Tse \& Ginneken (2017) en el que el grupo EFI obtuvo un rendimiento superior que EFE y grupo control (C). No hubo diferencias significativas entre los grupos EFE y EFI en el estudio realizado con sujetos con alta experiencia (Schorer, Jaitner, Wollny, Fath \& Baker, 2012).

En aquellas tareas en las que se desarrollaban las habilidades de lanzamiento de jabalina, de disco y de bolos (Asadi, Abdoli, Farsi \& Saemi, 2014; Zarghami, Saemi \& Fathi, 2012; Abdollahipour et al., 2015), el grupo EFE obtuvo mejores resultados, siendo el nivel de pericia de los participantes bajo.

Se realizaron nueve estudios en los que se diseñaron tareas en las que se evaluaba la precisión de los participantes en la ejecución de un put en golf desde diferentes distancias, obteniendo el grupo EFE ventajas significativas en el 75\% de las pruebas realizadas. En el estudio realizado por Perkins-Ceccato, Passmore \& Lee (2003), los golfistas altamente cualificados se desempeñaron mejor con instrucciones de atención externa que con instrucciones de enfoque interno, en contraste con los golfistas de baja destreza, que se desempeñaron mejor con el enfoque interno. Por otra parte, Poolton, Maxwell, Masters \& Raab (2006) llevaron a cabo dos experimentos. En el primero de ellos no hubo diferencias significativas entre los grupos durante las pruebas de aprendizaje y rentención, al contrario que ocurriera bajo una carga de tarea secundaria, en la que el grupo EFE se mantuvo más sólido. En el segun- 


\begin{tabular}{|c|c|c|c|c|}
\hline Estudio (Id.) & Tarea, diseño del estudio & Sujetos/Grupos & Nivel & Resultados \\
\hline $\begin{array}{l}\text { Lohse, Jones, Healy \& Sherwood } \\
\text { (2014b) }\end{array}$ & Dardos: lanzamiento de dardos; 4 sesiones, 300 ensayos & 15 (no se indica edad); $\mathrm{EFI}_{1}, \mathrm{EFI}_{2}, \mathrm{EFE}_{1}, \mathrm{EFE}_{2}, \mathrm{C}$ & B & EFE $>$ EFI, C \\
\hline $\begin{array}{l}\text { Marchant, Clough \& Crawshaw } \\
\text { (2007) }\end{array}$ & Dardos: lanzamiento de dardos a una diana; 1 sesión, 50 ensayos & 67 (20 años); EFI, EFE, C & B & $\mathrm{EFE}, \mathrm{C}>\mathrm{EFI}$ \\
\hline \multirow{2}{*}{$\begin{array}{c}\text { Lohse, Sherwood \& Healy (2014a) } \\
\text { Estudio } 1 \\
\text { Estudio } 2 \\
\end{array}$} & $\begin{array}{l}\text { Dardos: lanzamiento de dardos a una diana. Idem con un peso } \\
\text { añadido a la muñera de } 1 \mathrm{~kg} ; 1 \text { sesión, } 67 \text { ensayos }\end{array}$ & 40 (no se indica edad); EFI, EFE & B & EFE>EFI \\
\hline & $\begin{array}{l}\text { Dardos: lanzamiento de dardos a una diana. Idem con un peso } \\
\text { añadido a la muñera de } 1 \mathrm{~kg} ; 3 \text { sesiones, } 198 \text { ensayos }\end{array}$ & 48 (no se indica edad); EFI, EFE & B & $\mathrm{EFE}>\mathrm{EFI}$ \\
\hline Emmanuel, Jarus \& Bart (2008) & Dardos: lanzamiento de dardos; 2 sesiones, 70 ensayos & Jóvenes 34 (9.04 años); adultos 32 (28,73 años); EFI, EFE & B & $\begin{array}{l}\text { Jóvenes } \\
\text { EFE=EFI } \\
\text { Adultos } \\
\text { EFE }>\text { EFI }\end{array}$ \\
\hline Lohse, Sherwood \& Healy (2010) & $\begin{array}{l}\text { Dardos: lanzamiento de dardos a 2,3m de la diana; } 1 \text { sesión, } 21 \\
\text { ensayos }\end{array}$ & 12 (no se indica edad); EFI, EFE & B & EFE>EFI \\
\hline $\begin{array}{l}\text { Marchant, Clough, Crawshaw \& } \\
\text { Levy (2009) }\end{array}$ & Dardos: lanzamiento de dardos; 2 sesiones, 60 ensayos & 72 (19.82 años); EFI, EFE & B & EFE $>$ EFI \\
\hline $\begin{array}{l}\text { Schorer, Jaitner, Wollny, Fath \& } \\
\text { Baker (2012) }\end{array}$ & $\begin{array}{l}\text { Dardos: lanzamiento de dardos a 2,37m de la diana; } 1 \text { sesión, } 84 \\
\text { ensayos }\end{array}$ & 6(33.2 años); EFI, EFE & $\mathrm{B} / \mathrm{A}$ & EFE >EFI \\
\hline Tse \& Ginneken (2017) & Dardos: lanzamiento de dardos; 1 sesión, 50 ensayos & 60 (10.4 años); EFI, EFE, C & B & $\begin{array}{l}\text { Alto control consciente: } \mathrm{EFI}>\mathrm{EFE}, \mathrm{C} \text {; } \\
\text { bajo control consciente: } \mathrm{EFE}>\mathrm{EFI}>\mathrm{C}\end{array}$ \\
\hline $\begin{array}{l}\text { Asadi, Abdoli, Farsi \& Saemi } \\
\text { (2014) }\end{array}$ & Lanzamiento de jabalina: 4 sesiones, 20 ensayos & 21 (23.5 años); EFI, $\mathrm{EFE}_{1}, \mathrm{EFE}_{2}$, C & B & $\mathrm{EFE}_{1}, \mathrm{EFE}_{2}>\mathrm{EFI}, \mathrm{C}$ \\
\hline Zarghami, Saemi \& Fathi (2012) & Lanzamiento de disco de $2 \mathrm{~kg}$; 3 sesiones, 45 ensayos & 20 (22 años); EFI, EFE & B & EFE>EFI \\
\hline $\begin{array}{l}\text { Abdollahipour, Wulf, Psotta \& } \\
\text { Palomo (2015) }\end{array}$ & $\begin{array}{l}\text { Bolos: lanzamiento de bolos con la mano dominante; } 1 \text { sesión, } 24 \\
\text { ensayos }\end{array}$ & 36 (8.5 años); EFI, EFE, C & B & EFE>EFI \\
\hline Land, Frank \& Schack (2014) & $\begin{array}{l}\text { Golf: mediante un put introducir la pelota en un hoyo situado a } \\
\text { distancias entre } 2-5 \mathrm{~m} .3 \text { sesiones, } 540 \text { ensayos. }\end{array}$ & 20 (26.8 años); EFI, EFE & B & EFE $>$ EFI \\
\hline Wulf, Lauterbach \& Toole (1999) & $\begin{array}{l}\text { Golf: mediante un put introducir la pelota en un hoyo situado a } \\
\text { distancias entre } 2-5 \mathrm{~m} .2 \text { sesiones, } 110 \text { ensayos. }\end{array}$ & 22 (21-29 años); EFI, EFE & B & EFE $>$ EFI \\
\hline $\begin{array}{l}\text { Poolton, Maxwell, Masters \& Raab } \\
\text { (2006) } \\
\text { Estudio } 1\end{array}$ & $\begin{array}{l}\text { b Golf: mediante un put introducir la pelota en un hoyo situado a una } \\
\text { distancia de } 2 \mathrm{~m} \text {. Idem realizado un conteo de los tonos altos; } 2 \\
\text { sesiones, } 360 \text { ensayos }\end{array}$ & 30 (24.1 años); EFI, EFE & $\mathrm{B} / \mathrm{A}$ & $\begin{array}{l}\text { Tarea simple: EFE=EFI; Tarea doble: } \\
\text { EFE>EFI }\end{array}$ \\
\hline Estudio 2 & $\begin{array}{l}\text { Golf: mediante un put introducir la pelota en un hoyo situado a una } \\
\text { distancia de } 2 \mathrm{~m} \text {. Idem realizado un conteo de los tonos altos; } 2 \\
\text { sesiones, } 360 \text { ensayos }\end{array}$ & 39 (20.4 años); EFI, EFE & $\mathrm{B} / \mathrm{A}$ & $\begin{array}{l}\text { Tarea simple: EFE=EFI; Tarea doble: } \\
\text { EFE>EFI }\end{array}$ \\
\hline $\begin{array}{l}\text { Wulf \& Su (2007) } \\
\quad \text { Estudio } 1\end{array}$ & $\begin{array}{l}\text { Golf: mediante un put introducir la pelota en un hoyo situado a una } \\
\text { distancia de } 15 \mathrm{~m} \text {; } 1 \text { sesión, } 60 \text { ensayos }\end{array}$ & 30 (no se indica edad); EFI, EFE, C & B & EFE $>$ EFI, C \\
\hline Estudio 2 & $\begin{array}{l}\text { Golf: mediante un put introducir la pelota en un hoyo situado a una } \\
\text { distancia de } 15 \mathrm{~m} \text {; } 1 \text { sesión, } 60 \text { ensayos }\end{array}$ & 6 (no se indica edad); EFI, EFE, C & A & $\mathrm{EFE}>\mathrm{EFI}, \mathrm{C}$ \\
\hline Bell \& Hardy (2009) & $\begin{array}{l}\text { Golf: mediante un put introducir la pelota en un hoyo situado a una } \\
\text { distancia de } 20 \mathrm{~m} \text {; } 1 \text { sesión, } 30 \text { ensayos }\end{array}$ & 33 (37.06 años); EFI, EFE & A & EFE $>$ EFI \\
\hline An, Wulf \& Kim (2013) & $\begin{array}{l}\text { Golf: mediante un put introducir la pelota en un hoyo situado a una } \\
\text { distancia de } 4 \mathrm{~m} ; 1 \text { sesión, } 100 \text { ensayos }\end{array}$ & 24 (27.3 años); EFI, EFE, C & B & $\mathrm{EFE}>\mathrm{EFI}, \mathrm{C}$ \\
\hline $\begin{array}{c}\text { Perkins-Ceccato, Passmore \& Lee } \\
(2003)\end{array}$ & $\begin{array}{l}\text { Golf: mediante un put introducir la pelota en un hoyo situado a una } \\
\text { distancia de } 10 \text { a } 24 \mathrm{~m} ; 1 \text { sesión, } 80 \text { ensayos }\end{array}$ & 20 (no se indica edad); EFI, EFE & $\mathrm{B} / \mathrm{A}$ & $\begin{array}{l}\text { (B) EFI }>\text { EFE } \\
\text { (A) EFE }>\text { EFI }\end{array}$ \\
\hline $\begin{array}{l}\text { Saemi, Porter, Wulf, Ghotbi- } \\
\text { Varzaneh \& Bakhtiari (2013) }\end{array}$ & $\begin{array}{l}\text { Tenis: golpear la pelota con la raqueta para acertar a una diana } \\
\text { colocada a } 3 \mathrm{~m} ; 1 \text { sesión, } 180 \text { ensayos }\end{array}$ & 20 (10.1 años); EFI, EFE & B & EFE $>$ EFI \\
\hline $\begin{array}{c}\text { Winkelman, Clark \& Ryan (2017) } \\
\text { Estudio } 1\end{array}$ & Atletismo: esprint 10 metros; 1 sesión, $6 \times 10$ metros & 17 (19.41 años); EFI, EFE, C & B & $\begin{array}{c}\text { Tiempo: } \\
\text { EFE, C>EFI } \\
\text { Variables cinéticas } \\
\text { EFE }=\text { EFI }=\mathrm{C} \\
\end{array}$ \\
\hline Estudio 2 & Atletismo: esprint 10 metros; 1 sesión, $6 \times 10$ metros & 13 (28 años); EFI, EFE, C & A & $\begin{array}{c}\text { Tiempo: } \\
\mathrm{EFE}=\mathrm{EFI}=\mathrm{C} \text { Variables cinéticas } \\
\mathrm{EFE}=\mathrm{EFI}=\mathrm{C}\end{array}$ \\
\hline $\begin{array}{l}\text { Porter, Wu, Crossley \& Knopp } \\
\text { (2015) }\end{array}$ & Atletismo: esprint 20m; 3 sesiones, 9 ensayos & 84 (20.17 años); EFI, EFE, C & B & $\mathrm{EFE}>\mathrm{EFI}, \mathrm{C}$ \\
\hline $\begin{array}{l}\text { Schücker, Hagemann, Strauss \& } \\
\text { Volker (2009) }\end{array}$ & Atletismo: carrera continua; 2 sesiones, 33 minutos & 24 (30.8 años); EFI, EFE & A & EFE $>$ EFI \\
\hline $\begin{array}{l}\text { Schücker, Anheier, Hagemann, } \\
\text { Strauss \& Volker (2013) }\end{array}$ & Atletismo: Carrera continua; 1 sesión, $3 \times 10$ minutos & 20 (29.05 años); EFI, EFE, C & A & $\mathrm{EFE}>\mathrm{EFI}, \mathrm{C}$ \\
\hline $\begin{array}{c}\begin{array}{c}\text { Schücker, Schmeing \& Hagemann } \\
\text { (2016b) }\end{array} \\
\end{array}$ & Atletismo: carrera continua; 1 sesión, 24 minutos & 30 (12.84 años); $\mathrm{EFI}_{1}, \mathrm{EFI}_{2}, \mathrm{EFE}, \mathrm{C}$ & B & EFE $>$ EFI \\
\hline $\begin{array}{l}\text { Flores, Child \& Chiviacowsky } \\
\text { (2015) }\end{array}$ & Ciclismo: pedalear durante 10 metros, 1 sesión, 20 ensayos & 108 (6-10 años); $\mathrm{EFE}_{1}, \mathrm{EFE}_{2}$, EFI, C & B & $\mathrm{EFE}_{1}, \mathrm{EFE}_{2}>\mathrm{EFI}, \mathrm{C}$ \\
\hline $\begin{array}{c}\text { Schücker, Fleddermann, de } \\
\text { Lussanet, Elischer, Bohmer \& } \\
\text { Zentgraf (2016a) }\end{array}$ & $\begin{array}{l}\text { Ciclismo: pedaleo encima de una bicicleta de carrera; } 1 \text { sesión, } 49 \\
\text { minutos }\end{array}$ & 25 (28.4 años); $\mathrm{EFI}_{1}, \mathrm{EFI}_{2}, \mathrm{EFI}_{3}, \mathrm{EFE}$ & M & $\mathrm{EFE}>\mathrm{EFI}_{1}, \mathrm{EFI}_{2}, \mathrm{EFI}_{3}$ \\
\hline Totsika \& Wulf (2003) & $\begin{array}{l}\text { Ciclismo: pedaleo encima de una bicicleta de carrera durante } 7 \mathrm{~m} ; 1 \\
\text { sesión, } 20 \text { ensayos }\end{array}$ & 22 (23.4 años); EFI, EFE & B & EFE $>$ EFI \\
\hline Parr \& Button (2009) & Remo; 24 sesiones, 6 semanas & EFI, EFE, Distal-EFE, Proximal-EFE, C & B & $\begin{array}{l}\text { EFE }>\text { EFI, Distal EFE }>\text { Proximal } \\
\text { EFE }>C\end{array}$ \\
\hline Stoate \& Wulf (2011) & Natación: 1 sesión, 3 largos modalidad craw. & 30 (17.5 años); EFI, EFE, C & A & EFE, C>EFI \\
\hline $\begin{array}{l}\text { Maxwell \& Masters (2002) } \\
\text { Estudio } 1\end{array}$ & $\begin{array}{l}\text { Tarea de equilibrio: mantener el equilibrio sobre un tablero de } \\
100 \mathrm{~cm} \text { de diámetro. Idem mientras se cuentan tonos altos; } 1 \text { sesión, } \\
\underline{16 \text { ensayos }}\end{array}$ & 20 (21.20 años); EFI, EFE & B & EFE=EFI \\
\hline Estudio 2 & $\begin{array}{l}\text { Tarea de equilibrio: mantener el equilibrio sobre un tablero de } \\
100 \mathrm{~cm} \text { de diámetro. Idem mientras se cuentan tonos altos; } 1 \text { sesión, } \\
16 \text { ensayos }\end{array}$ & 20 (22.16 años); EFI, EFE & B & EFE=EFI \\
\hline Wulf, Höß \& Prinz (1998) & $\begin{array}{l}\text { Esqui: realizar movimientos oscilatorios encima de un simulador } \\
\text { de slalom de esquí; } 3 \text { sesiones, } 90 \text { ensayos }\end{array}$ & 33 (25 años); EFI, EFE, C & B & $\mathrm{EFE}>\mathrm{C}>\mathrm{EFI}$ \\
\hline $\begin{array}{l}\text { Lawrence, Gottwald, Hardy \& Khan } \\
\qquad \text { (2011) }\end{array}$ & Gimnasia: secuen & 40 (20.3 años); EFI, EFE, C & B & $\begin{array}{c}\text { Fase adquisición } \\
\text { EFI }>\text { EFE, } C \\
\text { Fase retención y transferencia } \\
\text { EFE }=E F I=C \\
\end{array}$ \\
\hline
\end{tabular}

Notas: EFE grupo estrategia de focalización externa; EFI grupo estrategia de focalización interna; C grupo control; DT doble tarea; B bajo; M moderado; A alto.

do experimento fueron ambos grupos los que mostraron una degradación de su rendimiento bajo la carga de una tarea secundaria.

Sólo se realizó un estudio en el que los participantes debían golpear una pelota con una raqueta de tenis para acertar a una diana situada a 3 metros de distancia (Saemi, Porter, Wulf, Ghotbi-Varzaneh \& Bakhtiari, 2013). De nuevo, el grupo EFE mostró un mejor rendimiento. En otros estudios, se les solicitaba a los sujetos que realizaran sprints y carreras continuas, registrándose el tiempo empleado en recorrer una determinada distancia, algunas variables cinemáticas y la economía del esfuerzo durante la carrera. En el 83\% de las pruebas el grupo EFE fue más rápido. Schücker, Hagemann, Strauss \& Volker (2009), Schücker,
Anheier, Hagemann, Strauss \& Volker (2013) y Schücker, Schmeing \& Hagemann (2016b) demostraron también que una focalización de la atención centrada en los efectos del movimiento (EFE) favorece la mejora en la economía de funcionamiento durante la carrera, en base a los registros realizados de consumo máximo de oxígeno, frecuencia cardíaca, lactato en la sangre y un cuestionario sobre el esfuerzo subjetivo percibido (RPE).

Pero en el estudio realizado por Winkelman, Clark \& Ryan (2017), el grupo EFE sólo mostró un mayor rendimiento en el primer experimento, realizado con sujetos con nivel de pericia bajo. En el segundo experimento, los sujetos con alta cualificación obtuvieron similar rendi- 
miento independientemente de la focalización adoptada durante la ejecución de las tareas.

En los estudios donde se realizaron tareas relacionadas con el ciclismo (Flores, Child \& Chiviacowsky, 2015; Schücker, Fleddermann, de Lussanet, Elischer, Bohmer \& Zentgraf(2016a); Totsika \& Wulf, 2003), el remo (Parr \& Button, 2009) y la natación (Stoate \& Wulf, 2011), de nuevo el grupo EFE obtuvo un mejor desempeño. En otros estudios se diseñaron tareas en las que se analizaba el efecto de un enfoque externo de la atención durante una tarea de equilibrio sobre una tabla, mostrándose más beneficioso en algunos casos (Wulf et al., 1998), y en otros el mismo rendimiento que el grupo EFI, no deteriorándose con la ejecución de una tarea secundaria de conteo de tonos altos y bajos (Maxwell \& Masters, 2002).

Lawrence, Gottwald, Hardy \& Khan (2011) analizaron la ejecución técnica de una secuencia de movimientos en gimnastas nóveles. Durante la fase de adquisición el grupo EFI rindió a mayor nivel, pero en las pruebas de retención y transferencia los grupos EFE y EFI mostraron similar desempeño.

Finalmente, en líneas generales los protocolos verbales realizados en los estudios demostraron que el grupo interno acumula durante las tareas más conocimiento que el grupo externo.

\section{Discusión}

El objetivo principal fue realizar una revisión sistemática de la literatura para determinar el efecto de una EFE y EFI sobre el aprendizaje y el rendimiento de una habilidad deportiva individual. Se analizaron un total de 37 estudios que cumplían con los criterios de inclusión establecidos inicialmente. Se ha utilizado una EFE a través de la focalización de la atención en los efectos de la ejecución de una habilidad, con objeto de favorecer su control automático, evitando con ello la interrupción de los procesos inconscientes durante su ejecución. En el $71 \%$ de las pruebas realizadas el grupo EFE obtuvo mejores resultados, tal y como ocurriera en las investigaciones realizadas por Perkos, Theodorakis \& Chroni (2002), Zachry et al. (2005) y Reeves et al. (2007), que afirman que focalizar la atención de manera externa durante la adquisición de una habilidad favorece su eficacia y generalización, pues mejora los procesos automáticos, al contrario que ocurriera con los grupos EFI, cuyas instrucciones conducen al procesamiento de una cantidad mayor de información, generando con ello una sobrecarga de la memoria de trabajo, y por tanto es más susceptible a degradar su rendimiento.

Pero en otros estudios, sin embargo, es el grupo de EFI el que muestra mayor rendimiento (Perkins-Ceccato et al., 2003), o se obtienen los mismos resultados independientemente de la estrategia de focalización utilizada (Emmanuel et al., 2008; Schorer et al., 2012; Winkelman et al., 2017; Maxwell \& Masters, 2002; Lawrence et al., 2011).

Los estudios realizados con grupos de diferentes edades (Emmanuel et al., 2008; Perkins-Ceccato et al., 2003) refuerzan los hallazgos de otros estudios con respecto a los beneficios de una EFE en los adultos (Zachry et al., 2005; Saemi, Abdoli, Farsi \& Sanjari, 2016), al contrario que ocurriera con los jóvenes. Bernstein (1996) afirma que esto es debido a que los sujetos con más edad tienen un mayor nivel de automatización de los movimientos corporales, siendo éste un proceso implícito o intuitivo más desarrollado en los adultos en el que el movimiento se vuelve más eficiente y la regulación más precisa. En estas edades una EFI puede interrumpir el proceso de control motor que regula de forma automática los movimientos. Los jóvenes, al contrario, al no tener todavía suficientemente desarrollados estos mecanismos incidentales, pueden utilizar las instrucciones orientadas hacia el propio movimiento como guía para la mejora de su aprendizaje.

De igual manera, en los estudios donde se realizaba una intervención en grupos con nivel de pericia baja y alta, los grupos EFI con baja experiencia mostraron mejor desempeño (Poolton et al., 2006; PerkinsCeccato et al., 2003), pues el aprendizaje intencional de las reglas explicitadas les ha podido servir como mecanismo de detección y co- rrección de errores que facilite una mejora adicional en la ejecución de la habilidad, favoreciendo la continuidad en la búsqueda de hipótesis acerca de la ejecución de dicho movimiento, al contrario que ocurriera con los sujetos con experiencia alta, corroborando las conclusiones de Beilock et al. (2002) de que la atención a componentes relevantes de la habilidad es perjudicial para el rendimiento en jugadores de alta calificación. Tal y como afirman Schmidt \& Lee (1999), una vez que una habilidad se automatiza no requiere atención consciente, lo cual sugiere que el desempeño de las tareas motrices se vuelve más automatizado con la práctica y la experiencia, y por consiguiente menos exigente. Centrar por tanto la atención en los detalles de una habilidad bien aprendida puede interrumpir su correcta ejecución.

Sin embargo, en una tarea de esprint de diez metros, los gruposEFE y EFI muestran similar rendimiento en sujetos con alta experiencia (Winkelman et al., 2017). Estos resultados evidencian la necesidad de tener en cuenta el tipo de habilidad a ejecutar, tal y como concluyen autores como Lawrence et al., (2011) tras la realización de una intervención en la que los sujetos debían ejecutar una rutina gimnástica y ambas condiciones de focalización mejoraron su rendimiento, afirmando que el tipo de habilidad requerida era multifacética en comparación con otras investigaciones en las que se requería la ejecución de un movimiento discreto (Lohse et al., 2010; Saemi et al., 2013; Wulf \& Su 2007).

En el estudio realizado por Poolton et al., (2006), los participantes debían realizar una tarea principal simple que consistía en ejecutar un put de golf, con objeto de introducir una pelota en un agujero situado a una distancia de dos metros. No hubo diferencias significativas entre los grupos EFE y EFI. Sin embargo, cuando realizaban la misma actividad de forma simultánea a una tarea secundaria (conteo de tonos), es el grupo EFE el que desempeñaba mejor esta labor. Esto puede ser debido a que cuando se realizaba únicamente la tarea principal, ésta no suponía una sobrecarga atencional para ninguno de los grupos, siendo suficiente la capacidad de la memoria de trabajo, al contrario que ocurriera durante la ejecución simultánea de ambas tareas, en la que se hace evidente las diferencias de rendimiento entre ambos grupos. Wulf, McNevin \& Shea (2001) afirman que un enfoque externo demanda menor atención que un enfoque interno, y por tanto permite disponer de mayores recursos para la ejecución de la habilidad.

Estos resultados indican que la dificultad de la tarea puede ser un factor potencial clave en el resultado de los estudios. No obstante, en el estudio realizado por Maxwell \& Masters (2002) en el que se diseñó una tarea de equilibrio, los grupos EFE y EFI no degradan su rendimiento en condiciones de doble tarea, deduciéndose de los cuestionarios post-experimentales que el grupo EFI cambió hacia un modo de EFE a pesar de las instrucciones recibidas, debido a la percepción personal de la ineficacia del enfoque inicial adoptado. Autores como Albaret \& Thon (1998) afirman que si la tarea a aprender es compleja, los sujetos podrían haber recurrido a un procesamiento cognitivo más profundo, realizando adaptaciones perceptivas-cognitivas independientemente del tipo de estrategia empleada inicialmente durante el proceso de enseñanza-aprendizaje, desarrollando patrones de comportamiento que hagan frente a los escenarios complejos de las tareas. Por tanto, tal y como afirman Maxwell, Masters \& Eves (2000), la predisposición a adoptar un enfoque u otro particular puede depender de la naturaleza de la habilidad requerida.

Basándonos en la afirmación que realizan Lam, Maxwell \& Masters (2009a) de que la degradación del rendimiento es el resultado de la cantidad de información que se procesa en la memoria de trabajo, del esfuerzo realizado en la tarea y del tipo de información procesada por el sujeto, debemos tener también en cuenta que en algunos estudios que se han realizado que tratan de mostrar los beneficios de la atención externa frente a la interna se suministra a los grupos experimentales diferentes cantidades de información, recibiendo generalmente los sujetos inducidos a adoptar una focalización interna más información que los sujetos inducidos hacia una focalización externa (Lam, Maxwell \& Masters, 2009b), siendo los resultados en la mayor parte de los casos favorables a estos últimos. Sólo hemos encontrado unos pocos estudios que han tenido en cuenta esta particularidad, equilibrando entre ambos grupos la 
cantidad de información suministrada, obteniendo de nuevo en algunos casos resultados favorables a la focalización externa (Marchant, Clough \& Crawshaw, 2007; Wulf et al., 1998), y en otros resultados similares para ambos grupos (Maxwell \& Masters, 2002). Poolton et al. (2006) concluyen tras un estudio en el que desarrollaron tareas relacionadas con el golf, que la interrupción de la actuación del enfoque interno de los participantes fue consecuencia de la acumulación de un gran número de información explícita (sobrecarga atencional), y no en cambio de la propia focalización interna per se, siendo por tanto cuestión de la cantidad de información y no de la calidad, pues tal y como afirman (Lam et al., 2009b), la carga atencional asociada con el procesamiento de una única regla es menor que la carga atencional asociada con el procesamiento de varias reglas.

\section{Conclusión}

Si bien el reducido número de estudios analizados en nuestra revisión, la heterogeneidad de sus diseños y los resultados obtenidos no nos permite realizar conclusiones precisas que podamos generalizar a otros grupos, sí existen evidencias de ciertas tendencias que a continuación pasamos a exponer. En líneas generales podemos afirmar que reducir la cantidad de información que se le suministra a los sujetos beneficia el aprendizaje y la retención de una habilidad deportiva, ya que la carga atencional disminuye considerablemente, disponiendo con ello de mayores recursos para la ejecución de la habilidad, por lo que la provisión de un gran número de instrucciones no será necesario para la adquisición de algunas habilidades motrices. Centrar la atención hacia los detalles de una habilidad bien aprendida para controlar los movimientos interrumpe su correcta ejecución, pero en cambio puede ser beneficioso para jugadores con baja experiencia. Además, una información orientada hacia los efectos del movimiento (EFE) obtiene mayores ventajas que una orientada hacia los movimientos del cuerpo durante la ejecución (EFI). Finalmente, las variables tipo de habilidad, grado de dificultad, edad y nivel de experiencia de los participantes son factores potencialmente moderadores de los resultados, por lo que deberán ser analizados para evaluar el efecto de una EFE y EFI.

\section{Limitaciones}

Los resultados mencionados anteriormente se deberían analizar con cautela, ya que no podemos afirmar que la estrategia seguida por los jugadores en este tipo de tareas haya sido únicamente EFE o EFI, pues todas las medidas realizadas para llegar a estas conclusiones han sido tomadas de forma indirecta. En algunos estudios la cantidad de información ofrecida a los grupos de participantes ha sido diferente, con lo que se aconseja tener en cuenta dicha premisa en ulteriores intervenciones. En todos los estudios se ha empleado un diseño de intervención transversal, lo cual reduce considerablemente el número de ensayos realizados por los participantes, y por consiguiente, dificulta el establecimiento de una relación causa-efecto entre las variables analizadas, ya que el conocimiento no se fija en el momento del aprendizaje, sino que se estabiliza y desarrolla con el tiempo, por lo que se propone la realización de estudios de corte longitudinal. Para finalizar, el aprendizaje está sujeto no sólo a la mera interpretación y acumulación de información, sino también a una variedad de influencias emocionales que pueden transmitirse a través de la retroalimentación suministrada, pudiendo dicha valencia dificultar el aprendizaje, con lo que aconsejamos realizar una análisis multidimensional en futuros estudios. Además, debemos garantizar que el diseño de las sesiones de entrenamiento sea realmente representativa de la competición real, para lo cual sería fundamental identificar cuáles son los factores o condiciones de práctica que confieren un carácter específico a la tarea, cual debe ser la duración, la frecuencia y la regularidad de las sesiones, así como los procesos de entrenamiento, con objeto de favorecer que se produzcan adaptaciones estables y significativas, como en los estudios realizados por Reeves et al. (2007) y Turner \& Martinek (1999).

\section{Aplicaciones prácticas}

Se propone realizar pruebas de retención que determinen la consistencia en el tiempo de ciertas actitudes desarrolladas bajo este tipo de estrategia, como las realizadas por Poolton, Masters \& Maxwell (2007). Así mismo, se podrían realizar pruebas de transferencia en situaciones reales de competición, con la intención de analizar los efectos del aprendizaje de dicha estrategia, como las desarrolladas por Raab (2002), además de analizar el efecto que tiene el diseño de este tipo de sesiones (duración, frecuencia, regularidad, combinación de variables, etc), con objeto de determinar la carga de trabajo necesaria para que se produzcan en los sujetos adaptaciones estables y significativas, favoreciendo con ello la optimización de nuestras sesiones prácticas.

\section{Referencias}

Abdollahipour, R., Wulf, G., Psotta, R., \& Palomo M. (2015). Performance of gymnastics skill benefits from an external focus of attention. Journal of Sports Sciences, 33(17), 1807-1813.

Al-Abood, S.A., Bennett, S. J., Hernandez, F.M., Ashford, D., \& Davids, K. (2002). Effect of verbal instructions and image size on visual search strategies in basketball free throw shooting. Journal of Sports Sciences, 20(3), 271-278.

Albaret, J.M., \& Thon, B. (1998). Differential effects of task complexity on contextual interference in a drawing task. Acta Psychologica, 100(1-2), 9-24.

An, J., Wulf, G., \& Kim, S. (2013). Increased carry distance and X-factor stretch in golf through an external focus of attention. Journal of Motor Learning and Development, 1(1), 2-11.

Asadi, A., Abdoli, B., Farsi, A., \& Saemi, E. (2014). Effect of various attentiona focus instructions on novice javelin throwing skill performance. Medicina Dello Sport, 67(4), 1-7.

Baumeister, R.F.(1984). Choking under pressure: Self-consciousness and paradoxical effects of incentives on skillful performance. Journal of Personality and Social Psychology, 46(3), 610-620.

Baumeister, R.F., \& Showers, C.J. (1986). A review of paradoxical performance effects: Choking under pressure in sports and mental tests. European Journal of Social Psychology, 16(4), 361-383.

Beilock, S.L., y Carr, T.H. (2001). On the fragility of skilled performance: What governs choking under pressure? Journal of Experimental Psychology: General, 130(4), 701-725.

Beilock, S.L., Carr, T.H., MacMahon, C., \& Starkes, J.L. (2002). When paying attention becomes counterproductive: Impact of divided versus skill-focused attention on novice and experienced performance of sensorimotor skills. Journal of Experimental Psychology: Applied, 8(1), 6-16.

Bell, J.J., \& Hardy, J. (2009). Effects of attentional focus on skilled performance in golf. Journal of Applied Sport Psychology, 21(2), 163-177.

Bernstein, N.A. (1996). Dexterity and its development. In: Latash M.L, Turvey M.T (eds). On Dexterity and Its Development. Mahwah, NJ: Erlbaum, 171204

Brocken, J.E. A., Kal, E.C., \& Van der Kamp, J. (2016). Focus of attention in children's motor learning: Examining the role of age and working memory. Journal of Motor Behavior, 48(6), 527-534.

Emanuel, M., Jarus, T., \& Bart, O. (2008). Effect of focus of attention and age on motor acquisition, retention, and transfer:Arandomized trial. Physical Therapy, 88(2), 251-260

Flores, F., Child, J.F., \& Chiviacowsky, S. (2015). Benefits of external focus instructions on the learning of a balance task in children of different ages. International Journal of Sport Psychology, 46(4), 311-320.

Fuchs, A.H. (1962). The progression-regression hypothesis in perceptual-motor skill learning. Joumal of Experimental Psychology, 63(2), 177-182.

González, G, Valdivia-Moral, Cachón, Zurita \& Romero-Ramos (2017). Influencia del control del estrés en el rendimiento deportivo: la autoconfianza, la ansiedad y la concentración en deportistas. Retos, 32, 3-6.

Holfelder, B., \& Schott, N. (2014). Relationship of fundamental movement skills and physical activity in children and adolescents:Asystematic review.Phychology of Sport and Exercise, 15(4), 382-391.

Ioannidis, J.P., Patsopoulos, N.A., \& Rothstein, H.R. (2008). Reasons or excuses for avoiding meta-analysis in forest plots. British Medical Journal, 336(7658), 1413-1415.

Jackson, R.C., Ashford, K.J., \& Norsworthy, G. (2006). Attentional focus, dispositional reinvestment, and skilled motor performance under pressure. Journal of Sport \& Exercise Psychology, 28(1), 49-68.

Kais, K., \& Raudsepp, L. (2005). Intensity and direction of competitive state anxiety, self-confidence and athletic performance. Kinesiology, 37(1), 13-20.

Lam, W.K., Maxwell, J.P., \& Masters, R.S.W. (2009a). Analogy learning and the 
performance of motor skills under pressure. Journal of Sport \& Exercise Psychology, 31(3), 337-357.

Lam, W.K., Maxwell, J.P., \& Masters, R.S.W. (2009b). Analogy versus explicit learning of a modified basketball shooting task: Performance and kinematic outcomes. Journal of Sports Sciences, 27(2), 179-191.

Land, W., Frank, C., \& Schack, T. (2014). The influence of attentional focus on the development of skill representation in a complex action. Psychology of Sport and Exercise, 15(1), 30-38.

Lawrence, G.P., Gottwald, V.M., Hardy, J., \& Khan, M.A. (2011). Internal and external focus of attention in a novice form sport. Research Quarterly for Exercise and Sport, 82(3), 431-441.

Liao, C.M., \& Masters, R.S.W. (2001). Journal of Sports Sciences, 19(5), $307-$ 319.

Lohse, K., Sherwood, D., \& Healy, A. (2010). How changing the focus of attention affects performance, kinematics, and electromyography in dart throwing. Human Movement Science, 29(4), 542-555.

Lohse, K., Sherwood, D., \& Healy,A. (2014a). On the advantage of an external focus of attention: A benefit to learning or performance? Human Movement Science, 33(1), 120-134.

Lohse, K., Jones, M., Healy, A., \& Sherwood, D. (2014b). The role of attention in motor control. Journal of Experimental Psychology: General, 143(2), 930 948.

Marchant, D.C., Clough, P.J., \& Crawshaw, M. (2007). The effects of attentional focusing strategies onnovice dart-throwing performanceand their task experiences. International Journal of Sport and Exercise Psychology, 5(3), 291-303.

Marchant, D.C., Clough, P.J., Crawshaw, M., \& Levy, A. (2009). Novice motor skill performance and task experience is influenced by attentional focusing instructions and instruction preferences. International Journal of Sport and Exercise Psychology, 7(4), 488-502.

Masters, R.S.W., Polman, R.C.J. y Hammond, N.V. (1993). Reinvestment: A dimension of personality implicatedinskill breakdownunderpressure.Personality and Individual Diferences, 14(5), 655-666.

Masters, R.S.W., Poolton, J.M., Maxwell, J.P., \& Raab, M. (2008). Implicit Motor Learning and Complex Decision Making in Time-Constrained Environments. Journal of Motor Behavior, 40(1), 71-79.

Maxwell, J.P. Masters, R.S.W., \& Eves, F.F. (2000). From novice to no knowhow: a longitudinal study of implicit motor leaming. Journal of Sports Sciences, 18(2), 111-120.

Maxwell, J.P., \& Masters, R.S.W.(2002). External versus internal focus instructions: is the learner paying attention? International Journal of Applied Sports Sciences, 14(2), 70-88.

Maynard, I.W., \& Howe, B.L. (1987). Interrelations of trait and state anxiety with game performance of rugby players. Perceptual and Motor Skills, 64(2), 599602.

Moher D, LiberatiA, Tetzlaff J,Altman D. G The PRISMA Group (2009). Preferred Reporting Items for Systematic Reviews and Meta-Analyses: The PRISMA Statement. PLoS Med 6(7), 1-6.

Newell, K. M. (1986). Constraints on the development of coordination. In M.G Wade y H.T.A. Whiting (Eds.), Motor development in children: aspect of coordination and control (pp. 341-360). Nijhoff: Dordrecht.

Núñez, A. \& García, A. (2017). Relación entre el rendimiento y la ansiedad en el deporte: una revisión sistemática. Retos, 32, 172-177.

Parr, R., \& Button, C. (2009). End-point focus of attention: Learning the 'catch' in rowing. International Journal of Sport Psychology, 40(4), 616-635

Perkins-Ceccato, N., Passmore, S.R., \& Lee, T.D. (2003). Effects of focus of attention depend on golfers' skill. Journal of Sport Sciences, 21(8), 593-600.

Perkos, S., Theodorakis, Y., \& Chroni, S. (2002). Enhancing performance and skill acquisition in novice basketball players with instructional self-talk. Sport Psychologist, 16(4), 368-383.

Poolton, J.M., Masters, R.S.W. \& Maxwell, J.P. (2007). Passing thoughts on the evolutionary stability of implicit motor behaviour: Performance retention under physiological fatigue. Consciousness and Cognition, 16(2), 456-468.

Poolton, J.M., Maxwell, J.P., Masters, R.S.W., \& Raab, M. (2006). Benefits of an external focus of attention: Common coding or conscious processing? Journal of Sports Sciences, 24(1), 89-99.

Porter, J.M., Wu, W.F.W., Crossley, R.M., \& Knopp, S.W. (2015). Adopting an external focus of attention improves sprinting performance in low-skilled sprinters. Journal of Strength and Conditioning Research, 29(4), 947-953.

Raab, M. (2002). T-ECHO: Model of decision making to explain behaviour in experiments and simulations under time pressure. Psychology of Sport \& Exercise, 3(2), 151-171.

Raab, M. (2003). Decision making in sports: Influence of complexity on implicit and explicit learning. Journal of Sport and Exercise, 1, 310-337.

Reeves, J.L., Tenenbaum, G, \& Lidor, R. (2007). Choking in front of the goal: The effects of self-consciousness training. International Journal of Sport and Exercise Psychology,5(3), 240-254.
Saemi, E., Porter, J.M., Wulf, G, Ghotbi-Varzaneh, A., \& Bakhtiari, S. (2013). Adopting an external focus facilitates motor learning in children with attention deficit and hyperactivity disorder. Kinesiology, 45(2), 179-185.

Saemi, E., Abdoli, B., Farsi, A., \& Sanjari, M.A. (2016). The interaction of external/internal and relevant/irrelevant attentional focus on skilled performance: the mediation role of visual information. Medicina dello Sport. Rivista di Fisiopatologia dello Sport, 70(4), 419-429.

Sánchez, R., \& Sebastián, M.A. (2015). Attention alters appearances and solves the «many-many problem»: implications for research in skill acquisition and execution. European Journal of Human Movement, 34, 156-179.

Schmidt, R.A. \& Lee, T.D. (1999). Motor control and learning: A behavioral emphasis. Champaign, IL: Human Kinetics.

Schoenfeld, B., Vigotsky,A. Contreras, B. \& Golden, S. (2018). Differential effects of attentional focus strategies during long-term resistance training. European journal of Sport Science, 18(5), 1-8.

Schorer, J., Jaitner, T., Wollny, R., Fath, F., \& Baker, J. (2012). Influence of varying focus of attention conditions on dart throwing performance in experts and novices. Experimental Brain Research, 217(2), 287-297.

Schücker, L., Anheier, W., Hagemann, N., Strauss, B., \& Volker, K. (2013). On the optimal focus of attention for efficient running at high intensity. Sport, Exercise and Performance Psychology, 2(3), 207-219.

Schücker, L., Fleddermann, M., de Lussanet, M., Elischer, J., Bohmer, C., \& Zentgraf, K. (2016a). Focusing attention on circular pedaling reduces movement economy in cycling. Psychology of Sport \& Exercise, 27, 9-17.

Schücker, L., Hagemann, N., Strauss, B., \& Volker, K. (2009). The effect of attentional focus on running economy. Journal of Sport Sciences, 27(12), 12421248.

Schücker, L., Schmeing, L., \& Hagemann, N. (2016b). «Look around while running!» Attentional focus effects in inexperienced runners. Psychology of Sport and Exercise, 37, 205-2012.

Smith, M. (2010). Research methods in sport. UK: Learning Matters.

Stoate, I., \& Wulf, G. (2011). Does the attentional focus adopted by swimmers affect their performance? International Journal of Sport Science \& Coaching, 6(1), 99-108.

Totsika, V., \& Wulf, G (2003). The influence of external and internal foci of attention on transfer to novel situations and skills. Research Quarterly Exercise and Sport, 74(2), 220-225.

Tse, A., \& Ginneken, W. (2017). Children's conscious control propensity moderates the role of attentional focus in motor skill acquisition. Psychology of Sport and Exercise, 31, 35-39.

Turner, A.P. y Martinek, T.J. (1999). An investigation into teaching games for understanding: Effects on skill, knowledge, and game play. Research Quarterly For Exercise and Sport 70(3), 286-296.

Winkelman, N., Clark, K., \& Ryan, L. (2017). Experience level influences the effect of attentional focus on sprint performance. Human Movement Science, 52, 84 95.

Wulf, G. (2013). Attentional focus and motor learning: a review of 15 years. International Review of Sport and Exercise Psychology, 6(1), 77-104.

Wulf, G. (2015). An external focus of attention is a conditio sine qua non for athletes: a response to Carson, Collins, and Toner (2015). Journal of Sports Sciences, 34(13), 1293-1295.

Wulf, G., Chiviacowsky, S., Schiller, E., \& Gentilini, L.T. (2010). Frequent external-focus feedback enhances motor learning. Frontiers in Psychology, 1,17.

Wulf, G., Höß, M., \& Prinz, W. (1998). Instructions for motor learning: Differential effects of internal versus external focus of attention. Journal of Motor Behavior, 30(2): 169-179.

Wulf, G., Lauterbach, B., \& Toole, T.(1999). The learning advantages of an external focus of attention in golf. Research Quarterly for Exercise and Sport, 70(2), 120-126.

Wulf, G., McNevin, N.H., \& Shea, C.H. (2001). The automaticity of complex motor skill learning as a function of attentional focus, The Quarterly Journal of Experimental Psychology, 54A(4), 1143-1154

Wulf, G., Shea, C.H., \& Park, J.H. (2001). Attention in motor learning: preferences for and advantages of an external focus. Research Quarterly for Exercise and Sport, 72(4), 335-344.

Wulf, G, \& Su, J. (2007). An external focus of attention enhances golf shot accuracy in beginners and experts. Research Quarterly for Exercise and Sport, 78(4), 384-389.

Zachry, T., Wulf, G., Mercer, J., \& Bezodis, N. (2005). Increased movement accuracy and reduced EMG activity as theresult of adopting an external focus of attention. Brain Research Bulletin, 67(4), 304-309.

Zarghami, M., Saemi, E., \& Fathi, I. (2012). External focus of attention enhances discus throwing performance. Kinesiology, 44(1), 47-51. 